\title{
Quem está motivado para aprender nos cursos oferecidos pelas empresas?
}

\author{
Luciana Mourão ${ }^{1}$ \\ Universidade Salgado de Oliveira, Rio de Janeiro-RJ, Brasil \\ Jesiane Marins \\ Universidade Estácio de Sá, Rio de Janeiro-RJ, Brasil
}

\begin{abstract}
Resumo: O mercado de trabalho tem se tornado mais competitivo e as organizações têm realizado programas de treinamento e desenvolvimento, buscando resultados eficazes em relação aos recursos humanos. Esta investigação teve como objetivo principal verificar se as características da clientela, do treinamento e das organizações influenciam nos resultados da motivação para aprender. A amostra foi obtida por conveniência, com 220 trabalhadores que estavam iniciando ações de capacitação em sete empresas privadas do Estado do Rio de Janeiro. Utilizaram-se escalas validadas para mensurar a motivação para aprender e as crenças sobre o sistema de treinamento. Foram realizadas análises descritivas e regressão múltipla padrão. Os resultados indicam que as crenças em relação ao sistema e tipo de treinamento, tipo de instrutor e tempo de empresa são preditoras da motivação para aprender. Foram discutidas implicações práticas dos resultados e sugerida agenda de pesquisa para a área.
\end{abstract}

Palavras-chave: motivação, crenças, treinamento.

\section{Who is motivated to learn in training offered by the companies?}

\begin{abstract}
With the growing competitiveness of the market, the organizations have made Training and Development Programs aims better results. This survey had as its main objective to verify if the individual, training and organizational characteristics influence the results of motivation to learn. The sample was of 220 workers who would be trained in seven private companies of the Rio de Janeiro. The scales to measure the motivation to learn and beliefs about the training were previously validated. There were descriptive and standard multiple regression analyses. The results indicate that the beliefs about training, the kind of training, the kind of instructor and the time of work are determinants of motivation to learn. We discussed practical implications of the results and suggested agenda to search for the area.
\end{abstract}

Keywords: motivation, beliefs, training.

\section{¿Quién está motivado para aprender en los cursos ofrecidos por las empresas?}

Resumen: El mercado laboral se ha vuelto más competitivo y las organizaciones han desarrollado programas de Capacitación y Desarrollo, con el fin de lograr resultados efectivos con relación a los recursos humanos. Esta investigación tuvo como objetivo principal verificar si las características del cliente, de la formación y de la organización influyen en el resultado de la motivación para aprender. La muestra fue de conveniencia, con 220 trabajadores que estaban empezando las acciones de formación, en siete empresas privadas en el estado de Rio de Janeiro. Se utilizó escalas validadas para medir la motivación para aprender y creencias sobre el sistema de formación. Fueron efectuados análisis descriptivo y regresión múltiple estándar. Los resultados indican que las creencias sobre el sistema de formación, el tipo de formación, el tipo de instructor y el tiempo de la empresa son predictores de la motivación para aprender. Fueron discutidas las repercusiones prácticas de las conclusiones y sugerido programa de investigación en el tema.

Palabras clave: motivación, creencias, capacitación.

Atualmente, muitas organizações estão aumentando os investimentos em capacitação e buscando criar seu diferencial competitivo a partir dos seus talentos internos. No entanto, se faz necessário compreender que o sucesso nas ações de Treinamento, Desenvolvimento e Educação - TD\&E - depende não só do interesse por parte das organizações, mas também da motivação para aprender por parte dos empregados. A esse respeito, Aguinis e Kraiger (2009) concluem que uma das formas de maximizar os benefícios do treinamento consiste em garantir que os

1 Endereço para correspondência:

Luciana Mourão. Universidade Salgado de Oliveira. Programa de Pósgraduação em Psicologia. Rua Marechal Deodoro, 263. Centro. CEP: 24.030-060. Niterói-RJ, Brasil. E-mail: mourao.luciana@gmail.com treinados estejam informados sobre o treinamento e motivados para o mesmo.

Pesquisa desenvolvida por Freitas e Borges-Andrade (2004) mostrou que as crenças sobre o sistema de TD\&E influenciam no impacto das ações de capacitação no trabalho. Ou seja, de acordo com a crença que o treinando tenha sobre o sistema, ele irá aplicar no trabalho com maior ou menor intensidade aquilo que aprendeu nos eventos de TD\&E. Porém, ainda não se investigou se as crenças sobre o sistema de TD\&E influenciam a motivação para aprender. Da mesma forma, há estudos correlacionando motivação para aprender com aprendizagem (Lacerda \& Abbad, 2003; Pilati, 2004; Warr \& Bunce, 1995). Mas o que leva ao aumento da motivação para aprender?

No Brasil, a motivação para aprender tem sido estudada mais como variável independente do que como variável 
dependente. Assim, conhece-se ainda pouco das variáveis que atuam como preditoras da motivação para o treinamento.

\section{Crenças sobre o sistema de TD\&E}

Neste estudo, considera-se que "as crenças são representações simbólicas, comumente baseadas em linguagens naturais, que tem sua origem na experiência pessoal e afirmam uma relação entre objetos concretos e abstratos, ou propriedades a eles atribuídas" (Krüger, 1995, p. 29). O pressuposto básico no estudo das crenças é o da sua organização lógica, subjetiva, em sistemas ou conjuntos logicamente estruturados, sendo capazes de ativar motivações e, portanto, condutas sociais influenciando por essa via processos coletivos (Krüger, 1995).

O estudo das crenças sobre o sistema de TD\&E se apóia em alguns dos pressupostos sobre crenças: (a) o fato de elas poderem ser compartilhadas; (b) ganharem força na medida em que mais pessoas compartilham da mesma crença; e (c) serem capazes de levar os indivíduos a mudanças. Portanto, é razoável supor que as crenças que uma pessoa tem sobre o sistema de TD\&E o possam influenciar a motivação para aprender em cursos de capacitação oferecidos pelas empresas.

Nas organizações, quando novos funcionários são contratados, os próprios colegas de trabalho começam a conversar sobre suas crenças acerca do Sistema de TD\&E daquela instituição, relatando, por exemplo, que o treinamento é algo importante naquela empresa, que ajuda a desenvolver atividades na prática e que é considerado nas promoções. Quando essas crenças são compartilhadas com esses novos empregados, boa parte dos novatos tomará essas falas como verdades e passarão a interpretar o treinamento naquela instituição da forma como lhe foi relatada pelos funcionários antigos, resultando em uma crença coletiva em relação ao treinamento.

Apesar do número reduzido de pesquisas realizadas sobre o assunto em questão, foram encontrados alguns resultados das investigações sobre crenças em relação ao treinamento e à aprendizagem. Freitas (2005) realizou uma pesquisa sobre o impacto de treinamento nos desempenhos do indivíduo e do grupo de trabalho e os relacionou com crenças sobre o sistema de treinamento e com o suporte à aprendizagem contínua.

Outro estudo realizado por Alencar (2005) teve como objetivo verificar as influências de crenças relacionadas ao trabalho no surgimento de sintomas na saúde dos trabalhadores e influências na produtividade. Os resultados demonstraram que foram obtidos dados qualitativos nas análises com associações significativas nas crenças e sugerem que a aprendizagem pode ser mais eficaz, quando os funcionários acreditam na importância e na necessidade do treinamento.

\section{Motivação para aprender}

$\mathrm{O}$ conceito de aprendizagem aplicado no âmbito das organizações tem uso pouco crítico ou sistemático (Coelho
Junior \& Borges-Andrade, 2008). Os autores apontam para a natureza multifacetada do conceito de aprendizagem e, a partir de uma análise conceitual do termo aprendizagem, esperam que o mesmo possa ser utilizado com maior sistematicidade em estudos organizacionais. Tomando por base as classificações apresentadas por Coelho Junior e BorgesAndrade (2008), é importante especificar que o presente artigo quando fala de "motivação para aprender" refere-se tão somente à aprendizagem formal, obtida quando uma pessoa participa de um programa de treinamento proporcionado pela organização onde trabalha.

A motivação para aprender é um tema que tem crescido e despertado interesse nos pesquisadores da área. Gagné (1985) aponta para a importância da motivação do aprendiz para que este atinja bons resultados de aprendizagem e de transferência para o trabalho em relação ao que foi aprendido. Segundo o autor, o estudo de motivação desperta elevado interesse entre os pesquisadores da Psicologia e o tema ainda está distante de ser completamente explicado, mas as evidências de que a motivação leva à aprendizagem na área educacional são abundantes.

Salanova, Hontangas e Peiró (1996, p.16) definem motivação como uma "ação dirigida a objetivos, sendo auto-regulada, biológica ou cognitivamente, persistente no tempo e ativada por um conjunto de necessidades, emoções, valores, metas e expectativas". Já Gondim e Silva (2004) apresentam quatro aspectos envolvidos no conceito de motivação: (a) ativação, considerado o estado inicial de estimulação em que se encontra a pessoa, no qual a mesma é capaz de desencadear a ativação, que pode localizar-se extrínseca ou intrinsecamente na pessoa; (b) direção que diz respeito ao objeto ou alvo de ação, que pode ser consciente ou inconsciente; (c) intensidade, considerada como variabilidade da força de ação e que pode diferir ao se admitir que a força depende de um estado anterior de carência (necessidade ou afeto) ou de um estado anterior a ser alcançado (alvo); e (d) persistência da ação, referindo-se à tentativa de compreender o fenômeno da motivação pela articulação entre a ativação, a direção e a intensidade da ação, atribuindo a sua manutenção a fatores pessoais (necessidades, desejos, traços de personalidade ou impulsos).

Diversas teorias foram desenvolvidas ao longo da história sobre os estudos em relação à motivação, porém no presente artigo serão destacados os estudos relacionados à motivação para aprender com enfoque nas organizações. A Teoria de Expectância de Vroom (1964) é uma das mais usadas em trabalhos acadêmicos que investigam os assuntos motivacionais. $\mathrm{O}$ autor desenvolveu a teoria dividida em três níveis: (a) Valência: grau de atração para o desempenho com o objetivo de alcançar a meta; (b) Instrumentalidade: visualização da relação entre a ação e a obtenção do resultado; e (c) Expectativa: antecipação dos resultados e probabilidade subjetiva. Essa teoria concebe a motivação fundamentalmente como uma força de natureza emocional e consciente, que é ativada no momento em que a pessoa é levada a escolher 
entre diversos planos de ação. A força dessa escolha estaria relacionada a dois fatores: expectativas individuais e avaliação subjetiva das conseqüências esperadas por meio da comparação entre várias alternativas de ação. Há um pressuposto de que as pessoas decidem sobre suas ações de modo instrumental, procurando maximizar seu prazer e seus ganhos e minimizar seu desprazer e suas perdas.

Focando especificamente organizações que valorizam o desenvolvimento e a aprendizagem dos seus funcionários, sendo o trabalho mental superior ao trabalho braçal, Gondim e Silva (2004) apresentam sete grupamentos englobando os principais aspectos motivacionais no trabalho. $\mathrm{O}$ primeiro aspecto motivacional diz respeito ao Ambiente de Trabalho, no qual os profissionais devem executar suas atividades com sucesso e, para isso, é necessário que a organização atenda algumas necessidades básicas no ambiente físico disponibilizado para o trabalho das pessoas. $\mathrm{O}$ segundo aspecto referese ao Gerenciamento de Pessoas, no qual o foco deve estar nas habilidades humanas na gestão de projetos. O terceiro, denominado Trabalho em Equipe, visa à criação e à manutenção de equipes, de forma que as pessoas tenham maior satisfação nas relações interpessoais e desempenhem seus limites máximos. O quarto aspecto - Sistema de Recompensas e Punições - deve ter como finalidade premiar os trabalhadores de modo tangível ou intangível por terem praticado comportamentos considerados desejáveis para a organização. $\mathrm{O}$ quinto aspecto, Desenvolvimento de Carreira, contempla os profissionais com treinamento, desenvolvimento e educação. Vale desatacar que este último tem relação com o presente estudo, embora aqui a motivação não seja variável-critério, mas possível preditora da aprendizagem e da reação a ações de TD\&E. O sexto aspecto, denominado Significado do Trabalho, pressupõe que quanto maior a identificação com as suas atividades, mais o profissional estará motivado a executá-las. E, por último, o aspecto Organização e Profissional envolve alguns aspectos motivacionais diretamente atribuídos à relação entre os valores organizacionais e os valores pessoais.

As teorias da Expectância e do Estabelecimento de Metas, são consideradas por Gondim e Silva (2004) como teorias de processo, sendo a motivação humana entendida como um processo de tomada de decisão em que estão em jogo as percepções, os objetivos, as expectativas e as metas pessoais. No presente estudo, será adotado como modelo teórico geral a Teoria da Expectância de Vroom (1964) sendo definida e pesquisada variável específica de motivação para aprender. Vale registrar que a Teoria da Expectância proporciona base racional e rica para a compreensão da motivação em determinado trabalho, oferece ampla possibilidade de aplicação e presta-se ao teste empírico (Muchinsky, 1994).

A respeito dos trabalhos empíricos sobre motivação para aprender, muitos têm buscado investigar a motivação em relação ao treinamento e relacioná-la com resultados do mesmo, como satisfação com o evento instrucional, aprendizagem, transferência do treinamento, entre outros
(Clark, Dobbins \& Ladd, 1993; Colquit, LePine e Noe, 2000; Holton, 1996; Seyler, Holton, Bates, Burnett e Carvalho, 1998; Tannenbaum, Cannon-Bowers, Salas e Mathieu, 1993; Warr e Bunce, 1995; Warr e Downing, 2000).

Na literatura nacional, Sallorenzo (2000) demonstra que a motivação do treinando após o treinamento tem uma relação maior com o resultado do treinamento em curto prazo, mas à medida que o treinado distancia-se do evento instrucional o resultado passa a ser cada vez mais controlado pelo ambiente de trabalho. A autora trabalhou com dados coletados por Abbad (1999), a partir de treinamentos realizados em um órgão de administração pública fechada. A amostra contemplada no estudo de Sallorenzo foi de 4.545 sujeitos, distribuídos em 227 cursos de curta duração em eventos de treinamento. Sallorenzo (2000) encontrou importante papel exercido pela motivação no resultado do treinamento em curto prazo (duas semanas), mas uma significativa perda de sua importância no resultado em longo prazo (três meses).

Colquit e cols. (2000) realizaram uma meta-análise das pesquisas na área, e construíram um modelo hipotético, o qual considera a motivação para aprender como preditora direta de resultados de aprendizagem e de transferência e desempenho no trabalho mediado por aprendizagem. Os autores relataram que as variáveis personalidade, idade e fatores situacionais têm relação de moderada a forte com motivação para aprender e também com a auto-eficácia pré-treinamento e a valência atribuída ao evento instrucional. A motivação nesse modelo seria preditora de alguns dos resultados de aprendizagem.

Em um outro estudo, Tharenou (2001) avaliou se a motivação para o treinamento, considerando a motivação para aprender e a motivação em função da expectativa de recompensas, explica a menor ou maior participação em ações de TD\&E. Para tal levantamento, utilizou 5.100 funcionários dos setores público e privado de comunidades australianas. A pesquisa foi composta por duas etapas, a primeira quando esses funcionários estavam em treinamento e a segunda 12 meses após o término dos cursos. Os resultados demonstraram que: (a) a motivação para o treinamento não é variável mediadora dos efeitos de suporte organizacional na participação das ações de TD\&E; (b) o suporte organizacional prediz mais a participação em ações de treinamento em empregados com alta motivação do que em empregados com baixa motivação; e (c) a motivação para o treinamento (tanto a motivação para aprender como a motivação relacionada à expectativa de recompensas futuras) explicam moderadamente a participação em ações de TD\&E.

Também na área organizacional, Tsai e Tai (2003) realizaram uma pesquisa que buscou investigar os fatores mediadores entre a atribuição e a motivação do treinamento, ou seja, se a importância percebida pelos empregados em relação ao programa de TD\&E seria uma variável que media o relacionamento entre a atribuição do treinamento e a motivação do mesmo. Os dados foram coletados com $184 \mathrm{em}-$ pregados que pertencem a 18 bancos. Aos participantes foi 
pedido que respondessem dois questionários: um no começo do programa de treinamento e o outro no meio. Os resultados suportaram a hipótese e mostraram que, comparado àqueles que participavam voluntariamente do treinamento, os que estavam no evento por imposição da chefia tiveram uma motivação mais elevada para a realização do mesmo. É, portanto, provável que, os empregados tendam a ficar mais motivados se perceberem que a organização demandará deles o que foi aprendido em determinado treinamento, ou seja, quando há maior percepção do valor instrumental daquela capacitação.

Uma pesquisa realizada recentemente foi a de Bell e Ford (2007), que teve como objetivo examinar os efeitos das reações dos treinandos, com a avaliação das habilidades voltadas para a motivação na aprendizagem. Eles desenvolveram um modelo que sugere três dimensões de reações da avaliação dos estagiários, distribuídas em justiça e utilidade, influência da motivação no treinamento e eficácia total do treinamento. O modelo foi testado usando uma amostra de 113 indivíduos, que estavam participando de um programa de treinamento com o objetivo de desenvolverem habilidades para dirigir caminhões e para prática da direção defensiva. $\mathrm{O}$ estudo tinha várias hipóteses entre elas: (a) as percepções de distribuições na justiça serão relacionadas positivamente a motivação para aprender, (b) as percepções dos treinandos sobre o serviço público serão relacionadas positivamente a motivação para aprender, e (c) a motivação dos trienandos para aprender estará relacionada positivamente às reações do treinamento, a auto-eficácia pós-treinamento, e intenções do comportamento. Essas três hipóteses de Bell e Ford (2007) foram confirmadas, permitindo concluir que a motivação para aprender está diretamente relacionada às impressões positivas que o indivíduo cria sobre o seu ambiente de trabalho e sobre o curso realizado, além de também de estar também relacionadas a características pessoais tais como auto-eficácia e intenções de comportamento.

Apesar do grande número de pesquisas realizadas sobre motivação em relação à aprendizagem, outros estudos são necessários com o objetivo de classificar as variáveis preditoras desse processo e investigar outras variáveis, tais como crenças sobre o sistema de TD\&E.

Nesse sentido, o presente estudo teve por objetivo conhecer o grau de motivação para aprender e verificar o poder de predição das variáveis relativas às características da clientela (crenças sobre o sistema de TD\&E e tempo de trabalho), características do treinamento (tipo de instrutor e tipo de treinamento) e características organizacionais (número de empregados) sobre a motivação para aprender.

\section{Modelo de Pesquisa}

O modelo do estudo tem como antecedentes as variáveis relativas às características da clientela, características do treinamento e características organizacionais e, como variável dependente, a motivação para aprender (Figura 1).

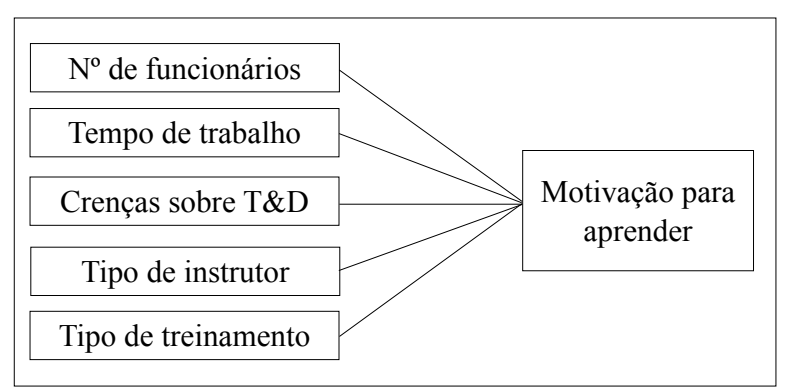

Figura 1. Modelo de pesquisa investigado

Em relação às definições operacionais e constitutivas das variáveis pesquisadas, compreende-se como definição constitutiva para crenças: "Crenças são proposições que, na sua formulação mais simples, afirmam ou negam uma relação entre dois objetos concretos ou abstratos, ou entre um objeto e algum atributo possível deste" (Krüger, 1995, p. 57). Já como definição operacional, foi adotada a escala sobre crenças em relação ao sistema de treinamento, desenvolvida e validada por Freitas e Borges-Andrade (2004).

Para a variável motivação para aprender a definição constitutiva é a apresentada por Colquit e cols. (2000, p. 678), que definem motivação para aprender como "direção, intensidade e persistência do comportamento orientado para a aprendizagem em contextos de treinamento". Já a definição operacional consiste na escala sobre motivação para aprender, desenvolvida e validada por Lacerda e Abbad (2003).

A hipótese geral para este estudo pode ser descrita da seguinte forma: Tempo de trabalho na empresa, número de funcionários da empresa, tipo de treinamento, tipo de instrutor e crenças em relação às ações de TD\&E são preditoras da motivação para aprender. Esperava-se que os funcionários com menor tempo de empresa, de empresas maiores, que vão participar de treinamentos de natureza comportamental, com instrutores externos e com crenças mais positivas sobre o sistema de TD\&E apresentem grau mais elevado de motivação para aprender.

As razões que levaram à hipótese de que pessoas com menor tempo de empresa apresentariam grau mais elevado de motivação para aprender derivam do fato de que funcionários mais recentes na organização costumam ter maior gama de conhecimentos para desenvolver e costumam estar menos acomodados. Por outro lado, esperava-se também que empresas maiores, com setores de treinamento e desenvolvimento mais estruturados, pudessem despertar maior motivação nos funcionários participantes dos cursos. Finalmente, esperava-se também que as crenças sobre o sistema de TD\&E influenciem a motivação para aprender, uma vez que tais crenças revelam o quanto os participantes dos cursos acreditam que ações de capacitação podem trazer resultados para os indivíduos e para as organizações nas quais eles trabalham. 


\section{Método}

\section{Participantes}

A amostra da pesquisa foi composta por 220 funcionários de sete empresas no Estado do Rio de Janeiro. A amostra foi obtida por conveniência e como o desenho da pesquisa exigia que o participante tivesse prestes a realizar uma ação de capacitação, não foi possível estender a pesquisa para um número maior de organizações. Em relação à escolha das empresas, optou-se por trabalhar apenas com organizações privadas, uma vez que as características do setor público e do setor privado podem influenciar os resultados da motivação para aprender e não se conseguiu um número de participantes que permitisse fazer essa comparação.

Um outro aspecto relevante no critério de inclusão das instituições foi que as empresas tivessem, entre os funcionários a serem capacitados, diversificação de graus de escolaridade, pesquisando-se trabalhadores com nível de ensino fundamental $(23,6 \%)$, médio $(63,8 \%)$ e superior $(12,4 \%)$. O perfil predominante dos participantes da pesquisa foi de homens $(59,1 \%)$, com experiências em treinamento em outras organizações $(63,6 \%)$, com mais de um ano de trabalho $(51,8 \%)$, idade média de 31 anos $(\mathrm{DP}=8,97)$ e salário na faixa de cinco salários mínimos.

Algumas análises foram conduzidas com o objetivo de verificar se o tamanho da amostra seria suficiente para garantir potência estatística aos testes que seriam realizados neste estudo. O software GPower (Faul, Erdfelder, Lang, \& Buchner, 2007) foi utilizado para as análises de potência. Verificou-se, por exemplo, que com um $N \geq 197$ seria possível detectar correlações relativamente fracas (iguais ou superiores que 0,25 ) considerando os parâmetros normalmente recomendados na literatura $(\alpha=0,05, \beta=0,05)$. Como os testes conduzidos envolveram amostras superiores a 200 , equivale dizer que a potência das análises esteve acima de 0,95 . Para facilitar uma melhor avaliação das inferências procurou-se relatar o tamanho do efeito observado. Ainda sobre a adequação do tamanho da amostra utilizou para uso da regressão as duas fórmulas de Green (1991) apresentadas por Tabachinick e Fidel (1996), sendo o tamanho da amostra adequado segundo as duas fórmulas (a primeira fórmula diz que a amostra mínima deve ser de $80+5$ vezes o número de variáveis independentes $(80+5 \times 7=115)$. A segunda fórmula postula que são necessários 111 sujeitos (104 + o número de variáveis independentes) para o teste de preditores individuais. Como a amostra foi de mais de 200 respondentes, esse critério foi atendido.

\section{Instrumentos}

Nesta pesquisa foi utilizado um questionário único que continha perguntas relativas aos dados demográficos e funcionais, além de duas escalas, uma relativa às crenças sobre o sistema de treinamento e outra relativa à motivação para aprender. O instrumento era auto-aplicável sendo o tempo médio de resposta de nove minutos.
A Escala de Crenças sobre o Sistema Treinamento desenvolvida por Freitas e Borges-Andrade (2004) possui 36 itens, associados a uma escala de 10 pontos, onde 1 = "Não Acredito" e 10 = "Acredito Totalmente". A Escala foi originalmente testada em uma amostra de aproximadamente 385 funcionários do Banco do Brasil e os autores identificaram a presença de três fatores: Fator 1 - Crenças sobre as contribuições do treinamento para o indivíduo e para a organização, Fator 2 - Crenças sobre o processo de levantamento de necessidades de treinamento e Fator 3 - Crenças sobre os resultados e o processo de treinamento. Os coeficientes de fidedignidade (Alfa de Cronbach) reportados para esses fatores foram $0,94,0,84$ e 0,82 , respectivamente. No presente estudo, foram utilizados os mesmos fatores sugeridos por Freitas e Borges-Andrade (2004), com coeficientes de fidedignidade semelhantes aos reportados no estudo original: $0,93,0,85$ e 0,87 , respectivamente.

A Escala de Motivação para Aprender desenvolvida originalmente por Warr, Allan e Birdi (1999) e traduzida para o português e analisada por Lacerda e Abbad (2003), permite identificar se os participantes se sentem motivados para aprender os conteúdos dados em programas de Treinamento. A versão original da Escala possui 6 itens associados a uma escala de sete alternativas de respostas variando de Discordo totalmente (1) a concordo totalmente (7). A validação no Brasil foi feita em uma amostra de 284 funcionários do Tribunal de Contas da União e as autoras identificaram uma estrutura unifatorial (Alfa de Cronbach $=0,75$ ), após descartar um dos seis itens que não compartilhou variância com o fator identificado. No presente estudo dois itens não compartilharam variância com a escala geral e foram descartados. O coeficiente de fidedignidade observado neste estudo para a escala foi de 0,77 .

\section{Procedimentos de coleta de dados}

O projeto de pesquisa foi aprovado pelo Comitê de Ética em Pesquisa da Universidade Salgado de Oliveira. A coleta de dados desta pesquisa foi realizada antes do início de ações de capacitação, na própria sala de aula de treinamento. $\mathrm{O}$ tempo de preenchimento foi livre. Os participantes assinaram o Termo de Consentimento Livre e Esclarecido e foi garantido a todos o direito de acesso aos resultados gerais da pesquisa e ao sigilo das informações individuais. A seleção dos participantes que responderam à pesquisa foi baseada na conveniência, tendo sido pesquisados os trabalhadores que iriam iniciar algum tipo de treinamento. Dentre esses, não foram feitas exclusões, pesquisando-se todos aqueles que as organizações encaminharam para as ações de treinamento.

\section{Procedimentos de análise dos dados}

A análise de dados foi feita utilizando-se o Software SPSS, versão 15.0. Foram realizadas as seguintes análises estatísticas: (a) análise de componentes principais/análise fatorial; (b) análises descritivas (média e desvio-padrão); (c) análises inferenciais (regressão múltipla padrão). 


\section{Resultados e Discussão}

Na primeira etapa da análise de dados foi feita a limpeza do banco de dados: identificação de dados omissos (missing values), examinadas as distribuições univariadas e bivariadas com o objetivo de avaliar normalidade, homogeneidade de variância e presença de dados extremos (outliers) univariados e multivariados (para esses últimos usando-se os parâmetros da Distância Mahalanobis). Algumas variáveis tiveram que ser transformadas. O critério numérico utilizado para avaliar a necessidade de transformações foi ancorado na simetria. Variáveis foram transformadas sempre que o escore $\mathrm{Z}$ para simetria (simetria/erro padrão de medida da simetria) foi maior que 1,96 (Hair, Anderson, Taham \& Black, 1992; Tabachnick \& Fidell, 1996). Foi necessário efetuar transformações nas seguintes variáveis: crenças sobre o sistema de TD\&E - fatores 1 e 3 - e motivação para aprender. Essas transformações resolveram satisfatoriamente os problemas de simetria, de curtose e de heterogeneidade de variância. As variáveis transformadas foram utilizadas em todas as análises de correlação e regressão. Para clarificar a interpretação dos resultados, são apresentadas, na Tabela 1, as médias e os desvios-padrão das variáveis de crenças antes da transformação (Tabachnick \& Fidell, 1996).

Para a regressão padrão linear também foram avaliadas, além da normalidade, a homogeneidade de variância, a multicolinearidade e a análise de resíduos. Em relação à multicolinearidade, encontrou-se alta correlação entre os fatores de crenças (coeficientes de Pearson de 0,63 a 0,74). Optou-se, assim, por trabalhar com a solução unifatorial para a escala trabalhando-se com uma média geral dos 36 itens para mensuração das crenças sobre o sistema de TD\&E. Os resultados obtidos com a aplicação do questionário de crenças sobre o sistema de treinamento, em termos de valores mínimos, máximos, média e desvio-padrão são apresentados na Tabela 1. A análise dos escores da escala de crenças sobre o Sistema de Treinamento permitiu concluir que os treinandos possuem uma crença positiva em relação às ações educacionais. Foi possível observar esse resultado a partir da média obtida tanto nos escores da escala geral, como nos escores dos três fatores: crenças sobre contribuições do treinamento, crenças sobre levantamento de necessidades e sobre resultados e crenças sobre os processos e os resultados do treinamento.

Tabela 1

Escores da escala de crenças e de seus fatores

\begin{tabular}{|c|c|c|c|c|}
\hline $\begin{array}{c}\text { Escala de Crenças - Escala Tipo } \\
\text { Likert (1 a 10) }\end{array}$ & Min & Max & $\mathbf{M}$ & DP \\
\hline $\begin{array}{l}\text { Crenças sobre o Sistema } \\
\text { Treinamento (Escala completa) }\end{array}$ & 2,44 & 9,83 & 7,90 & 1,17 \\
\hline $\begin{array}{l}\text { Crenças sobre contribuições do } \\
\text { treinamento (Fator 1) }\end{array}$ & & 10,00 & 8,16 & 1,18 \\
\hline $\begin{array}{l}\text { Crenças sobre levantamento de } \\
\text { necessidades (Fator 2) }\end{array}$ & 2,00 & 10,00 & 7,24 & 1,56 \\
\hline Crenças sobre resultados (Fator 3) & & 10,00 & 7,98 & 1,31 \\
\hline
\end{tabular}

A motivação para aprender foi mensurada por uma escala tipo Likert de sete pontos, sendo que quanto mais próximo de sete, maior a motivação e quanto mais próximo de um, menor a motivação para aprender. As respostas tiveram amplitude máxima, com respostas variando de um a sete, mas com uma média de 5,37 ( $\mathrm{DP}=0,71)$, indicando que os sujeitos pesquisados, antes de participarem dos cursos de capacitação apresentavam uma motivação para aprender de moderada a alta.

Para checar a hipótese geral, foi feita realizada uma regressão múltipla padrão, tendo a motivação para aprender como variável dependente e como variáveis independentes a crença sobre o sistema de TD\&E, o tempo de serviço (que teve que ser incluído como variável dummy - até um ano de trabalho e mais de um ano), tipo de treinamento (variável dummy - de natureza cognitiva e não-cognitiva), tipo de instrutor (variável dummy - interno e externo), e número de funcionários das empresas. A variável tempo de serviço foi transformada para variável dummy, uma vez que metade da amostra concentrou-se no primeiro ponto da escala (até um ano de serviço), o que acarretou problemas de normalidade e homogeneidade. Porém, como a variável era considerada importante e a literatura aponta que ela é preditora da motivação para aprender, optou-se por realizar a transformação para dummy e manter a sua inclusão no modelo de regressão.

Os resultados mostraram que apenas o número de funcionários da empresa não figurou como preditor da motivação para aprender, contudo, o poder preditivo do modelo foi baixo $\left(\mathrm{R}^{2}\right.$ ajustado $\left.=0,10\right)$, conforme pode ser visto na Tabela 2.

Tabela 2

Resultados da regressão com a VD motivação para aprender

\begin{tabular}{lccc}
\hline \multicolumn{1}{c}{ Variáveis } & B & $\mathbf{B}$ & $\mathbf{s r}^{2}$ \\
\hline Tempo de trabalho & $-0,004^{* *}$ & $-0,257$ & 0,001 \\
Tipo de instrutor & $0,055^{*}$ & 0,404 & 0,025 \\
Tipo de treinamento & $0,031^{*}$ & 0,203 & 0,016 \\
$\mathrm{~N}^{\mathrm{o}}$ funcionários & $-0,032$ & $-0,237$ & 0,022 \\
Crenças sobre T\&D & $0,001^{*}$ & 0,165 & 0,005 \\
\hline
\end{tabular}

Média $=5,37 \mathrm{DP}=0,71 \mathrm{~F}=3,91 \mathrm{t}($ constante $)=-5,54$

$\mathrm{R}=0,35 \mathrm{R}^{2}=0,12 \mathrm{R}^{2}$ ajust. $=0,10 * \mathrm{p}<0,05 ; * * \mathrm{p}<0,01$

Diante dos resultados apresentados é importante que os mesmos sejam discutidos tanto do ponto de vista de suas implicações como também em termos comparativos com achados da literatura da área. Na presente pesquisa foram altos os níveis de motivação para aprender para os funcionários das sete empresas pesquisadas. Esse resultado sinaliza que as pessoas estão interessadas em oportunidades que lhes permitam adquirir novas competências de trabalho. Ou seja, o cenário encontrado na revisão de literatura de que as organizações têm aumentado os seus investimentos em ações educacionais e reservado à área de Treinamento papel estratégico (Borges-Andrade, 2002; Marins, 2008; Mourão 
\& Borges-Andrade, 2005; Salas \& Cannon-Bowers, 2001) vai ao encontro também dos interesses individuais. Significa dizer que, tanto as empresas como os indivíduos estão valorizando ações de aprendizagem no ambiente organizacional.

A esse respeito, cabe considerar que esse interesse dos trabalhadores em aprender novas competências encontra aderência no conceito de empregabilidade, cada vez mais difundido na sociedade moderna. Segundo Aranha (2001, p. 281), "por empregabilidade entende-se a responsabilização do trabalhador pela obtenção e manutenção do seu emprego, através de um processo contínuo de formação e aperfeiçoamento." Portanto, na sociedade atual espera-se tanto o investimento das organizações em ações de capacitação como também a motivação dos empregados para aproveitar tais oportunidades de aprendizagem. Para o caso específico dos trabalhadores das sete empresas privadas do Estado do Rio de Janeiro, tal motivação para aprender foi confirmada pelos altos escores obtidos $(\mathrm{M}=5,37 ; \mathrm{DP}=0,71$; em uma escala de um a sete, onde quanto mais próximo de sete maior a motivação para aprender).

No que diz respeito à hipótese geral desta pesquisa (tempo de trabalho na empresa, número de funcionários da empresa, tipo de treinamento, tipo de instrutor e crenças em relação às ações de TD\&E são preditoras da motivação para aprender) foi quase totalmente confirmada, pois a análise de regressão múltipla padrão apontou que todas as variáveis atuaram como preditoras da motivação para aprender, à exceção do número de funcionários, como mostra a Figura 2. Porém, embora a regressão tenha indicado relações significativas entre a motivação para aprender e o tempo de trabalho, as crenças sobre o sistema de TD\&E, tipo de instrutor e tipo de treinamento o modelo apresentou baixo poder explicativo $\left(\mathrm{R}^{2}\right.$ ajustado $\left.=0,10\right)$.

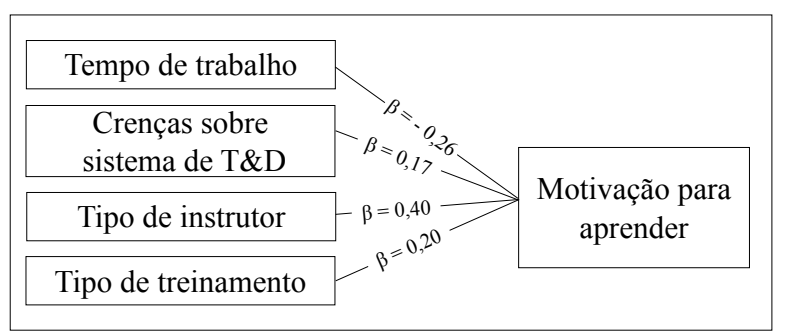

Figura 2. Modelo final para a variável motivação para aprender

Esse resultado pode ser explicado pelo fato de novos funcionários apresentarem, em geral, maior demanda por novos conhecimentos, habilidades e atitudes que permitam a sua adaptação às exigências do cargo naquela determinada organização. Vale destacar que uma série de estudos investigam se as características da clientela influenciam a motivação para aprender e o presente estudo confirma vários resultados previamente obtidos, sobretudo em pesquisas estrangeiras (Bell \& Ford, 2007; Colquit e cols., 2000; Tharenou, 2001; Tsai \& Tai, 2003). No que diz respeito ao tempo de trabalho, o resultado reitera alguns resultados anteriores como os de Pilati (2004), Bell e Ford (2007), Tsai e Tai (2003), os quais confirmam que os funcionários com menos tempo de trabalho apresentaram índices mais altos de motivação para aprender.

Em relação às crenças de treinamento, não se encontrou nenhum estudo em que as crenças aparecessem como preditoras da motivação para aprender, mas dois estudos (Freitas \& Borges-Andrade, 2004; Gonçalves, 2008) apontam que as crenças ajudam a explicar o impacto do treinamento no trabalho. Ou seja, a variável é importante para os resultados que se obtém dos cursos de capacitação que são oferecidos pelas organizações.

No que diz respeito ao tipo de treinamento, os resultados da pesquisa confirmam a hipótese de que treinamentos de natureza comportamental contribuem para aumentar a motivação para aprender. Acredita-se que os treinamentos de natureza comportamental despertam mais interesse porque eles permitem não apenas a capacitação específica para aquele tipo de trabalho, mas um desenvolvimento do participante como profissional num sentido mais amplo, caracterizando-se mais como ação de desenvolvimento, enquanto os meramente cognitivos costumam caracterizar-se como ações de treinamento. Nesse sentido, vale ressaltar o que explica Nadler (1984), para quem o desenvolvimento é um processo lento e gradativo que conduz ao conhecimento de si próprio e à realização de suas potencialidades. Ou seja, esse tipo de ação de capacitação que tem natureza comportamental, mesmo que apresente menor impacto do treinamento no trabalho (como descrito por Gonçalves, 2008), desperta maior interesse dos participantes por permitir-lhes o desenvolvimento de competências que podem ser utilizadas em situações diversas e não apenas em situações restritas de execução de tarefas de trabalho.

Em relação ao tipo de instrutor, os resultados da pesquisa confirmam a hipótese de que o fato de o instrutor ser externo contribui para aumentar a motivação para aprender. Possivelmente, os instrutores externos atraem mais a atenção dos participantes dos cursos pelo nome que muitos deles têm no mercado e pelo fato de que as pessoas tendem a apostar menos em instrutores internos que muitas vezes são caracterizados como "santo de casa" ou "solução caseira". Isso não significa, porém, que os instrutores internos tenham desempenho pior que os instrutores externos; apenas configura que, como salienta Block (1991), é difícil ser "um profeta em sua própria terra".

\section{Conclusão}

É cada vez mais comum encontrar nas organizações a demanda por processos de aprendizagem continuada, o que tem levado as organizações a investirem em ações de capacitação e buscarem compreender os seus resultados e como eles podem ser melhorados. Por outro lado, os trabalhadores também estão conscientes da importância do aperfeiçoamento 
contínuo, pois apresentam um grau elevado de motivação para aprender.

A presente pesquisa pretendeu enriquecer o debate sobre a influência de características da clientela (tempo de trabalho e crenças sobre TD\&E), de características do treinamento (tipo de treinamento e tipo de instrutor) e características organizacionais (número de funcionários) sobre a motivação para aprender. Para ampliar as possibilidades de interpretação dos resultados obtidos nessa pesquisa, os resultados da mesma foram comparados com a literatura da área.

No que se refere às crenças sobre o sistema de TD\&E, os resultados da presente pesquisa apontam crenças positivas por parte dos treinandos e indicaram que as crenças são preditoras da motivação para aprender. Também figuraram como preditoras da motivação, o tempo de trabalho, sendo que os mais novos apresentam mais motivação para aprender, o que reitera alguns resultados anteriores. Esse é um resultado que deve ser examinado com atenção pelos profissionais que atuam na área, pois pode sinalizar que o primeiro ano de cada funcionário é um período bastante propício para se investir em ações de capacitação, uma vez que os empregados tendem a apresentar maior motivação para aprender durante essa fase.

Também se concluiu que instrutores externos estão associados a uma maior motivação para aprender. Esses resultados indicam que as organizações podem selecionar com cuidado e divulgar mais seus instrutores internos a fim de que os mesmos possam despertar maior interesse dentre aqueles que irão participar dos cursos. Além disso, talvez fosse uma estratégia interessante mesclar o trabalho de instrutores internos e externos de maneira a atrair a atenção dos funcionários para as ações de capacitação e também "valoriza a prata da casa".

Entre as limitações deste trabalho encontra-se a amostra reduzida de organizações e a baixa variabilidade em termos de tempo de trabalho na instituição. O pequeno número de organizações para a pesquisa justifica-se, sobretudo, por duas razões: (a) o delineamento da pesquisa exigia que as pessoas pesquisadas tivessem iniciando uma ação de capacitação portanto, apenas organizações que se encontravam nessa fase poderiam fazer parte da pesquisa; (b) o universo de organizações que oferece capacitação para trabalhadores menos especializados (que tenham até o ensino médio) é menor e a pesquisa pretendia pesquisar vários tipos de trabalhadores e não apenas aqueles com escolaridade superior; e (c) muitas organizações receiam expor os dados relativos à motivação de seus funcionários, ainda que seja garantido o sigilo quanto aos dados dos participantes e o nome da organização. No que diz respeito à concentração de trabalhadores com pouco tempo de trabalho, este resultado ocorreu, sobretudo, por se ter incluído no estudo trabalhadores com escolaridade mais baixa - entre os quais há maior rotatividade.

Apesar dessas limitações, acredita-se que esta pesquisa pôde dar uma contribuição para as pesquisas da área, uma vez que foi realizada uma investigação ainda não encontrada em pesquisas no Brasil, testando se as crenças em relação ao treinamento são capazes de interferir positiva ou negativamente nos níveis motivação para aprender dos trabalhadores e se esse nível de motivação é afetado por outras variáveis como tempo de trabalho, número de funcionários da empresa, tipo de treinamento e tipo de instrutor.

Uma outra contribuição refere-se ao fato de o estudo incluir profissionais com diferentes níveis de escolaridade, com significativa presença de trabalhadores com escolaridade mais baixa (ensino fundamental e médio), uma vez que a maioria das pesquisas brasileiras sobre o tema costuma ser realizada com participantes predominantemente de nível superior.

Em relação a futuras pesquisas na área, sugere-se investigar não apenas a motivação dos treinandos para aprender, como também a motivação dos instrutores para ensinar, mensurando se professores motivados resultam em reações mais positivas em relação ao treinamento e em maior aprendizagem. Sugere-se também investigar se instrutores que se percebem mais motivados são ou não mais bem avaliados pelos treinandos. Ainda, uma outra proposta seria testar cursos com diferentes cargas horárias a fim de verificar se há maior ou menor motivação de acordo com a duração do curso.

Por fim, os resultados da presente pesquisa podem suscitar alguns questionamentos nos profissionais que atuam na área, de forma a instigar que eles revejam as suas práticas, tanto as relativas ao sistema de TD\&E como as afetas aos demais sistemas de gestão de pessoas. Assim, espera-se que o estudo possa ampliar a demanda por novas pesquisas sobre os temas investigados e contribuir para uma reflexão sobre o uso de achados empíricos nos processos de tomada de decisão nas organizações.

\section{Referências}

Abbad, G. S (1999). Um modelo integrado de avaliação do impacto do treinamento no trabalho - IMPACT. Tese de doutorado não publicada, Universidade de Brasília, Brasília, DF.

Aguinis, H., \& Kraiger, K. (2009). Benefits of training and development for individuals and teams, organizations, and society. Annual Review Psychology, 60, 451-474.

Alencar, M. D. C. D. (2005). Associações entre crenças relacionadas ao trabalho e suas influências na saúde dos trabalhadores e na produtividade, no setor de produção de frangos de corte: Uma abordagem ergonômica. Tese de doutorado não-publicada, Universidade Federal de Santa Catarina, Florianópolis.

Aranha, A. V. S. (2001). Formação profissional nas empresas: Locus privilegiado da educação do trabalhador? In S. M. Pimenta \& M. L. Côrrea (Orgs.), Gestão, trabalho e cidadania. Belo Horizonte: Autêntica.

Bell, B. S., \& Ford, J. K. (2007). Reactions to skill assessment: The forgotten factor in explaining motivation to learn. Human Resource Development Quarterly, 18(1), 33-62. 
Block, P. (1991). Consultoria: O desafio da liberdade. São Paulo: Makron, McGraw-Hill.

Borges-Andrade, J. E. (2002). Desenvolvimento de medidas em avaliação de treinamento. Estudos de Psicologia, 7(n. esp.), 31-43.

Clark, C. S., Dobbins, G. H., \& Ladd, R. T. (1993). Exploratory field study of training motivation: Influence of involvement, credibility, and transfer climate. Group \& Organization Management, 18, 292-307.

Coelho Junior, F. A., \& Borges-Andrade, J. E. (2008). Uso do conceito de aprendizagem em estudos relacionados ao trabalho e organizações. Paidéia (Ribeirão Preto), 18, 221-234.

Colquitt, J. A., LePine, J. A., \& Noe, R. A. (2000). Toward an integrative theory of training motivation: A meta-analytic path analysis of 20 years of research. Journal of Applied Psychology, 85, 678-707.

Faul, F., Erdfelder, E., Lang, A.-G., \& Buchner, A. (2007). $\mathrm{G}^{*}$ Power 3: A flexible statistical power analysis program for the social, behavioral, and biomedical sciences. Behavior Research Methods, 39, 175-191.

Freitas, I. A. (2005). Impacto de treinamento nos desempenhos do individuo e do grupo de trabalho: Suas relações com crenças sobre o sistema de treinamento e suporte à aprendizagem contínua. Tese de doutorado não-publicada, Instituto de Psicologia, Universidade de Brasília, Brasília, DF.

Freitas, I. A., \& Borges-Andrade, J. E. (2004). Construção e validação de escala de crenças sobre o sistema treinamento. Estudos de Psicologia, 9, 479-488.

Gagné, R. M. (1985). The conditions of learning and theory of instruction (4a ed.). New York: Holt, Rinchardt and Winston.

Gondim, S. M. G., \& Silva, N. (2004). Motivação no trabalho. In J. C. Zanelli, J. E. Borges-Andrade \& A. V. B. Bastos (Orgs.), Psicologia, organizações e trabalho. Porto Alegre: Artmed.

Gonçalves, A. I. P. (2008). Avaliação de treinamento: A expectativa e a reação do treinando como preditoras do impacto no trabalho. Dissertação de mestrado não-publicada, Universidade Salgado de Oliveira UNIVERSO, Niterói.

Green, B. L. (1991). Evaluating the effects of disasters. Journal of Consulting and Clinical Psychology, 3, 538-546.

Hair, J. F., Anderson, R. E., Taham, R. L., \& Black, W. C. (1992). Multivariate data analysis (3rd ed.). New York: MacMillian Co.

Holton, E. F. (1996). The flawed four-level evaluation model. Human Resource Development Quarterly, 7, 5-21.

Krüger, H. (1995). Psicologia das crenças: Perspectivas teóricas. Tese de concurso para professor titular nãopublicada, Universidade Estadual do Rio de Janeiro, Rio de Janeiro.
Lacerda, E. R. M., \& Abbad, G. (2003). Impacto do treinamento no trabalho: Investigando variáveis motivacionais e organizacionais como suas preditoras. RAC - Revista de Administração Contemporânea, 7(4), 77-96.

Marins, J. L. (2008). Avaliação de treinamento nos níveis de aprendizagem e reação: A influência da motivação para aprender e das crenças sobre o sistema de treinamento. Dissertação de mestrado não-publicada, Universidade Salgado de Oliveira - UNIVERSO, Niterói.

Mourão, L., \& Borges-Andrade, J. E. (2005). Avaliação de programas públicos de treinamento: Um estudo sobre o impacto no trabalho e na geração de emprego. Organizações \& Sociedade, 12(33), 13-38.

Muchinsky, P. M. (1994). Motivación laboral. In P. M. Muchinsky (Org.), Psicologia aplicada al trabajo: Una introduccion a la psicología industrial y organizacional (pp. 367 - 412). Bilbao: Desclée de Brouwer.

Nadler, L. (1984). The handbook of human resources development. New York: Wiley.

Pilati, R. (2004). Modelo de efetividade do treinamento no trabalho: Aspectos dos treinandos e moderação do tipo de treinamento. Tese de doutorado não-publicada, Instituto de Psicologia, Universidade de Brasília.

Salanova, M., Hontagas, P., \& Pieró, J. M. (1996). Motivation laboral. In J. M. Peiró \& F. Prieto (Eds.), Tratado de Psicologia del trabajo (Vol. 2). Madrid: Síntesis.

Salas, E., \& Cannon-Bowers, J. (2001). The science of training: Adecade of progress. Annual Review Psychology, 52, $471-499$.

Sallorenzo, L. H. (2000). Avaliação de impacto do treinamento no trabalho: Analisando e comparando modelos de predição. Dissertação de mestrado nãopublicada, Instituto de Psicologia, Universidade de Brasília, Brasília-DF.

Seyler, D. L., Holton, E. F., Bates, R. A., Burnett, M. F., \& Carvalho, M. A. (1998). Factors affecting motivation to transfer training. International Journal of Training \& Development, 2(1), 2-16.

Tabachinick, B. G., \& Fidell, L. S. (1996). Using multivariate statistics. New York: HarperCollins.

Tannenbaum, S. I., Cannon-Bowers, J. A., Salas, E., \& Mathieu, J. E. (1993). Factors that influence training effectiveness: A conceptual model and longitudinal analysis. Orlando: Naval Training Systems Center.

Tharenou, P. (2001). The relationship of training motivation to participation in training and development. Journal of Ocupational and Organizational Psychology, 74, 599-621.

Tsai, W-C., \& Tai, W-T. (2003). Perceived importance as a mediator of the relationship between training assignment and training motivation. Personnel Review, 32, 151-163.

Vroom, V. H. (1964). Work and motivation. New York: Wiley. 
Warr, P., Allan, C., \& Birdi, K. (1999). Predicting three levels of training outcome. Journal of Occupational and Organizational Psychology, 72, 351-375.

Warr, P., \& Bunce, D. (1995). Trainee characteristics and outcomes of open learning. Personnel Psychology, 48, 347-375.

Warr, P., \& Downing, J. (2000). Learning strategies, learning anxiety and knowledge acquisition. British Journal of Psychology, 91, 311-333.

Luciana Mourão é Professora Titular do Programa de Pósgraduação em Psicologia da Universidade Salgado de Oliveira, campus de Niterói.

Jesiane Marins é Mestre em Psicologia e Professora da Universidade Estácio de Sá.

Recebido: $15 / 10 / 2008$

$1^{a}$ revisão: $15 / 05 / 2009$

$2^{a}$ revisão: 02/10/2009

Aceite final: 04/11/2009 